\title{
Análise comparativa da cirurgia do buraco macular associada à remoção da membrana limitante interna com e sem coloração pela indocianina verde
}

\author{
Comparative analysis of macular hole surgery followed by internal limiting membrane \\ removal with and without indocyanine green staining
}

\author{
Oswaldo Moura Brasil ${ }^{1}$ \\ Oswaldo Ferreira Moura Brasil ${ }^{2}$
}

\begin{tabular}{|c|}
\hline RESUMO \\
\hline $\begin{array}{l}\text { Objetivo: Descrever comparativamente os resultados da cirurgia do bura- } \\
\text { co macular associada à remoção da membrana limitante interna com e sem } \\
\text { a coloração pela indocianina verde. Métodos: Foram avaliadas } 142 \text { cirur- } \\
\text { gias consecutivas de buraco macular com remoção de membrana limitante } \\
\text { interna realizadas no período de janeiro de } 2001 \text { a março de } 2004 \text {. Estas } \\
\text { foram divididas em dois grupos, baseados no uso ou não da coloração da } \\
\text { membrana limitante interna pela indocianina verde. Resultados: Os grupos } \\
\text { estudados foram semelhantes no que diz respeito ao perfil dos pacientes } \\
\text { e estágio pré-operatório dos buracos maculares. A acuidade visual pré- } \\
\text { operatóriamédiafoi igual a } 0,12 \pm 0,15 \text { no grupocom coloraçãoporindocianina } \\
\text { verde e } 0,18 \pm 0,18 \text { no grupo sem a coloração pela indocianina verde } \\
\text { (p=0,02). A acuidade visual pós-operatória média foi igual a } 0,27 \pm 0,27 \text { no } \\
\text { grupo com uso e } 0,43 \pm 0,25 \text { no grupo sem uso (p=0,0002). Observamos } \\
\text { melhora da acuidade visual em } 63 \% \text { dos casos no grupo com corante e em } \\
80,3 \% \text { dos casos no grupo sem corante. O fechamento do buraco macular } \\
\text { ocorreu em } 76,5 \% \text { dos casos com uso de indocianina verde e em } 95,1 \% \text { dos } \\
\text { casos sem utilização do corante. Conclusão: As cirurgias do buraco } \\
\text { macular com remoção da membrana limitante interna sem uso de corantes } \\
\text { apresentam melhores resultados visuais e anatômicos quando compara- } \\
\text { das àquelas com o auxílio da coloração pela indocianina verde. Recomen- } \\
\text { damos cautela ao utilizar a coloração com indocianina verde na cirurgia do } \\
\text { buraco macular pelo seu possível efeito tóxico. }\end{array}$ \\
\hline
\end{tabular}

Descritores: Mácula lútea; Perfurações retinianas/cirurgia; Mácula lútea/patologia; Verde de indocianina; Membrana basal/cirurgia; Estudo comparativo

Trabalho realizado no Setor de Retina e Vítreo do Instituto Brasileiro de Oftalmologia - IBOL.

${ }^{1}$ Chefe do Setor de Retina e Vítreo e Diretor do Instituto Brasileiro de Oftalmologia - IBOL - Rio de Janeiro (RJ) - Brasil.

${ }^{2}$ Médico do Setor de Retina e Vítreo do IBOL - Rio de Janeiro (RJ) - Brasil e Pós-graduando nível Doutorado pela Universidade Federal de São Paulo - UNIFESP São Paulo (SP) - Brasil.

Endereço para correspondência: Oswaldo Moura Brasil. Praia de Botafogo, 206 - Rio de Janeiro (RJ) CEP 22250-040

E-mail: ombrasil@uninet.com.br

Recebido para publicação em 15.09.2004

Versão revisada recebida em 20.05.2005

Aprovação em 15.11.2005

\section{INTRODUÇÃO}

No passado, a cirurgia para buraco macular (BM) era indicada apenas quando o buraco macular desencadeava um descolamento de retina ${ }^{(1)}$. Em 1991, a aplicação da cirurgia vitreorretiniana para tratamento do BM foi inicialmente descrita por Kelly e Wendel, tendo sido sua indicação confirmada e difundida mundialmente ${ }^{(2-3)}$.

A remoção da membrana limitante interna (MLI) aumenta significativamente o sucesso anatômico e visual na cirurgia do BM, mas pode ser um procedimento cirúrgico tecnicamente difícil de ser realizado ${ }^{(4-6)}$. Um dos principais fatores responsáveis pela dificuldade do procedimento é a identificação da MLI, pois a mesma é a primeira camada interna da retina, com uma espessura média de 1-3 micrômetros em pacientes não diabéticos.
\end{abstract}


Devido às dificuldades técnicas para a remoção da MLI, a utilização da indocianina verde (ICV) foi proposta para corar a MLI e conseqüentemente resultar em melhor detecção, bem como remoção mais segura da MLI, minimizando os riscos de dano à retina neurossensorial ${ }^{(7-8)}$.

Na nossa prática, após aproximadamente 300 cirurgias para BM, passamos a adotar a utilização da coloração da MLI com ICV rotineiramente. No entanto, após observarmos 2 casos de atrofia do nervo óptico em cirurgias sem intercorrências, resolvemos suspender o seu uso ${ }^{(9)}$.

O objetivo do nosso estudo foi avaliar comparativamente os resultados da cirurgia do buraco macular com remoção da membrana limitante interna com e sem a coloração pela indocianina verde, baseado em nossos últimos 142 casos.

\section{MÉTODOS}

Em uma série de 453 cirurgias consecutivas de BM, foram selecionadas, em análise retrospectiva, as últimas 142 cirurgias primárias para BM idiopático com remoção da MLI. As cirurgias foram realizadas na mesma instituição, pelo mesmo cirurgião e utilizando o mesmo sistema para vitrectomia Accurus $^{\circledast}$ 800DS (Dallas, EUA), com sonda Innovit ${ }^{\circledast}$ de 1800 cortes (Alcon, EUA), no período de janeiro de 2001 a março de 2004.

A técnica cirúrgica consistiu em realizar vitrectomia via pars plana com três esclerotomias localizadas a $4 \mathrm{~mm}$ do limbo nos olhos fácicos e $3 \mathrm{~mm}$ do limbo nos olhos pseudofácicos. Foi realizada a remoção do vítreo central seguida da apreensão por sucção da hialóide posterior, descolamento da mesma até região equatorial e sua remoção com o uso do vitreófago. A seguir, a MLI em torno do BM foi removida com utilização de micropinça tipo Eckardt (Dorc, Holanda). Em um grupo de pacientes (grupo A), a remoção da MLI foi efetuada após a coloração pela ICV a 2,5 mg/ml. Esta foi injetada sobre o pólo posterior após troca fluído-ar total e logo em seguida removida com troca ar-fluído para remoção da MLI. Posteriormente, foi efetuada troca fluído-gasosa com uso de C3F8 a 12,5\%. Em todos os casos, a solução de infusão utilizada foi o BSS plus ${ }^{\circledR}$ (Alcon, EUA) e a pressão intra-ocular foi mantida em $22 \mathrm{mmHg}$ com a linha de infusão forçada VGFI (Alcon, EUA). Em todos os casos, os pacientes foram orientados a adotar decúbito ventral pelo maior tempo possível na primeira semana de pósoperatório, com liberação progressiva do tempo de decúbito ventral até a reabsorção do gás.

As cirurgias foram divididas em dois grupos. $\mathrm{O}$ primeiro grupo, denominado A, consistiu das primeiras 81 cirurgias, sendo utilizada a coloração pela ICV em todos os procedimentos. Estas cirurgias ocorreram no período de janeiro de 2001 a fevereiro de 2002. O segundo grupo, denominado B, consistiu de 61 cirurgias, sem utilização da coloração por ICV, realizadas de março de 2002 a março de 2004.

Em ambos os grupos avaliamos: idade e sexo dos pacientes, olho operado, estágio pré-operatório do BM, acuidade visual pré e pós-operatória, uso da coloração pela ICV e fechamento do BM.
Em ambos os grupos, consideramos a acuidade visual, medida pela tabela de Snellen, entre o $2^{\circ}$ e $3^{\circ}$ mês do pós-operatório. Para fins de análise estatística, consideramos toda acuidade visual, seja pré ou pós-operatória, menor que 0,05 igual a zero.

A análise da significância estatística foi realizada pelo teste de Mann-Whitney/Wilcoxon para dois grupos. Consideramos estatisticamente significativo o valor de $\mathrm{p}<0,01$.

\section{RESULTADOS}

A idade variou de 35 a 79 anos, com média de $64,49 \pm 8,45$ no Grupo A e de 47 a 79 anos, com média de 65,04 $\pm 7,26$ no Grupo B. No Grupo A, 79,0\% dos casos eram do sexo feminino e $21,0 \%$ do sexo masculino e no Grupo B, $72,1 \%$ do sexo feminino e $27,9 \%$ do sexo masculino. Em relação ao olho operado, $59,3 \%$ eram direitos e $40,7 \%$ esquerdos no Grupo A, enquanto $45,9 \%$ eram direitos e $54,1 \%$ esquerdos no Grupo B (Tabela 1 ).

Na classificação do estágio pré-operatório, o estágio 2 representou 18,5\% dos casos no Grupo A e $23,0 \%$ dos casos no Grupo B, o estágio 3 representou 69,1\% dos casos no Grupo A e 68,9\% dos casos no Grupo B e o estágio 4 representou 12,3\% dos casos no Grupo A e 8,2\% dos casos no Grupo B (Tabela 2).

A acuidade visual pré-operatória média no Grupo A foi $0,12 \pm 0,15$ e no Grupo B $0,18 \pm 0,18$ ( $p=0,02)$. Estratificando por estágio pré-operatório, no Grupo A, a acuidade visual préoperatória média foi igual a $0,31 \pm 0,23$ no estágio $2,0,09 \pm 0,09$ no estágio 3 e $0,05 \pm 0,04$ no estágio 4 ( $p=0,0002$ ). No Grupo $B$, a acuidade visual pré-operatória média foi igual a $0,35 \pm 0,19$ no estágio $2,0,14 \pm 0,14$ no estágio 3 e $0,01 \pm 0,02$ no estágio 4 $(\mathrm{p}=0,0000)$.

A acuidade visual pós-operatória média no Grupo A foi $0,27 \pm 0,27$ e no Grupo B $0,43 \pm 0,25$ ( $p=0,0002)$. Estratificando por estágio pré-operatório, no Grupo $\mathrm{A}$, a acuidade visual pós-operatória média foi igual a $0,47 \pm 0,29$ no estágio 2 , $0,25 \pm 0,26$ no estágio 3 e $0,07 \pm 0,07$ no estágio 4 ( $p=0,0014)$. No Grupo B, a acuidade visual pós-operatória média foi igual a $0,64 \pm 0,24$ no estágio $2,0,37 \pm 0,22$ no estágio 3 e $0,35 \pm 0,23$ no estágio $4(\mathrm{p}=0,0032)$.

\begin{tabular}{|lcc|}
\hline \multicolumn{2}{|c|}{ Tabela 1. Perfil dos pacientes em relação aos Grupos A e B } \\
& Grupo A & Grupo B \\
Idade média & $64,46 \pm 8,45$ anos & $65,04 \pm 7,26$ anos \\
Sexo & $79,0 \%$ feminino & $72,1 \%$ feminino \\
& $21,0 \%$ masculino & $27,9 \%$ masculino \\
Olhos & $59,3 \%$ direitos & $45,9 \%$ direitos \\
& $40,7 \%$ esquerdos & $54,1 \%$ esquerdos \\
\hline
\end{tabular}

\begin{tabular}{|ccc|}
\hline \multicolumn{3}{c}{$\begin{array}{c}\text { Tabela 2. Distribuição do estágio pré-operatório do buraco macular } \\
\text { nos Grupos A e B }\end{array}$} \\
Estágio 2 & Grupo A & Grupo B \\
Estágio 3 & $18,5 \%$ & $23,0 \%$ \\
Estágio 4 & $69,1 \%$ & $68,9 \%$ \\
\hline
\end{tabular}




\begin{tabular}{|lcccc|}
\hline \multicolumn{4}{|c|}{ Tabela 3. Acuidades visuais médias pré e pós-operatórias, estratificadas pelo estágio pré-operatório do buraco macular, nos Grupos A e B } \\
& Grupo A pré & Grupo A pós & Grupo B pré & 0,35 \\
Estágio 2 & 0,31 & 0,47 & 0,14 & 0,64 \\
Estágio 3 & 0,09 & 0,25 & 0,01 & 0,37 \\
Estágio 4 & 0,05 & 0,07 & 0,18 & 0,35 \\
Média total & 0,12 & 0,27 & 0,43 \\
\hline
\end{tabular}

A tabela 3 mostra as acuidades visuais pré e pós-operatórias médias estratificadas por estágio pré-operatório do BM nos Grupos A e B.

A acuidade visual pós-operatória maior ou igual a 0,5 foi observada em $28 \%$ dos casos no Grupo A e em $44 \%$ dos casos no Grupo B.

Quando comparamos a acuidade visual pré-operatória com a pós-operatória observamos melhora da acuidade visual em 63,0\% dos casos no Grupo A e 80,3\% dos casos no Grupo B, acuidade visual igual em 18,5\% dos casos no Grupo A e 18,0\% dos casos no Grupo B e piora da acuidade visual em 18,5\% dos casos no grupo A e 1,6\% dos casos no Grupo B (Tabela 4).

O fechamento do BM ocorreu em $76,5 \%$ dos casos no Grupo A e 95,1\% dos casos no Grupo B. O Odds Ratio foi igual 5,92 (Intervalo de Confiança 95\%, p=0,001, teste exato de Fisher) quando avaliamos os casos do Grupo A, ou seja, a coloração pela ICV, como fator de risco para o BM aberto no pós-operatório (Tabela 5).

\section{DISCUSSÃO}

O sucesso da cirurgia do BM com remoção da MLI sem coloração pela ICV foi descrito diversas vezes na literatura. $\mathrm{O}$ índice de fechamento do BM foi observado em 92 a $100 \%$ dos $\operatorname{casos}^{(4-6,10)}$. A avaliação da melhora da acuidade visual em linhas na tabela de Snellen revelou melhora de pelo menos duas linhas em $85 \%$ dos olhos e de pelo menos três linhas em $77 \%$ dos casos, em diferentes estudos ${ }^{(4,10)}$. No nosso estudo, primeiro na literatura a comparar uma série de casos de remoção da MLI com e sem a coloração pela ICV realizados pelo

\begin{tabular}{|cccr|}
\hline \multicolumn{4}{|c|}{ Tabela 4. Resultado da comparação da acuidade visual pré-operató- } \\
ria com a pós-operatória nos Grupos A e B
\end{tabular}

\begin{tabular}{|lcc|}
\hline \multicolumn{3}{|c|}{$\begin{array}{l}\text { Tabela 5. Resultado anatômico da cirurgia do buraco macular nos } \\
\text { Grupos A e B }\end{array}$} \\
Buraco aberto & Buraco fechado \\
Grupo A & $23,5 \%$ & $76,5 \%$ \\
Grupo B & $4,9 \%$ & $95,1 \%$ \\
OR=5,92 (IC 95\%, p=0,001, teste exato de Fisher) \\
\hline
\end{tabular}

mesmo cirurgião, encontramos um índice de 95,1\% de fechamento e $80,3 \%$ de melhora da acuidade visual nas cirurgias do BM com remoção da MLI sem coloração pela ICV.

Tendo em vista os melhores resultados anatômicos da cirurgia do BM quando é removida a MLI, a coloração desta pela ICV foi proposta para facilitar o procedimento e descrita com bons resultados e ausência de toxicidade em alguns estudos ${ }^{(7-8,11-14)}$.

No entanto, tem sido cada vez mais frequiente a publicação de estudos propondo possíveis efeitos tóxicos da ICV, quando utilizada para corar a MLI. Foram descritos achados de acuidade visual pós-operatória desfavorável ${ }^{(15-18)}$, defeitos no campo visual com perda de visão periférica ${ }^{(15-17)}$ e anormalidades do epitélio pigmentário da retina ${ }^{(18-19)}$.

Em trabalho publicado em 2002, os autores não notaram melhora estatisticamente significativa da acuidade visual pósoperatória quando avaliaram 20 pacientes em que a ICV foi utilizada para corar a MLI. Notaram também defeitos no campo visual em $35 \%$ dos pacientes ${ }^{(15)}$.

Outros autores observaram que um grupo de 21 pacientes submetidos à remoção da MLI com coloração pela ICV não apresentou melhora significativa da acuidade visual e, ainda, $28,5 \%$ dos pacientes desenvolveram palidez do disco óptico e perda irreversível do campo visual periférico ${ }^{(16)}$.

Em outro estudo, ao comparar 18 pacientes em que foi utilizada a ICV com 39 pacientes sem seu uso, os autores relataram resultado visual significativamente menor com a administração intra-operatória da ICV e uma incidência de $50 \%$ de defeito no campo visual neste grupo ${ }^{(17)}$.

O fechamento do BM foi descrito em $86 \%$ de 21 pacientes submetidos à vitrectomia com remoção da MLI com coloração pela $\mathrm{ICV}^{(18)}$. Nestes casos, a acuidade visual pós-operatória média foi pior do que a pré-operatória. Foram detectadas alterações atróficas do epitélio pigmentário da retina em 47,6\% dos casos.

Outro trabalho também detectou anormalidades no epitélio pigmentário da retina em $27 \%$ dos casos de pacientes submetidos à remoção da MLI com coloração pela $\mathrm{ICV}^{(19)}$.

\section{CONCLUSÃO}

Encontramos em nosso estudo resultados amplamente favoráveis na remoção da MLI sem utilização da ICV, tanto no aspecto visual quanto anatômico. Além disso, podemos considerar a ICV como fator de risco para o insucesso anatômico do procedimento, ou seja, buraco aberto $(\mathrm{OR}=5,92, \mathrm{p}=0,001)$.

Acreditamos que, possivelmente, a ICV possua algum efei- 
to tóxico que possa explicar os resultados piores com o seu uso. Desta forma, recomendamos cautela ao indicar a utilização da coloração da MLI pela ICV na cirurgia do BM.

\section{ABSTRACT}

Purpose: To describe comparatively the outcomes of macular hole surgery followed by internal limiting membrane removal with and without indocyanine green staining. Methods: We evaluated 142 consecutive macular hole surgeries with internal limiting membrane removal performed in the period from January 2001 to March 2004. These were divided into two groups, based on the use or not of indocyanine green staining. Results: Both studied groups were similar concerning the patients' profile and preoperative stage of the macular holes. Mean preoperative visual acuity was $0.12 \pm 0.15$ in the group with indocyanine green staining and $0.18 \pm 0.18$ in the group without internal limiting membrane staining $(\mathrm{p}=0.02)$. Mean postoperative visual acuity was $0.27 \pm 0.27$ in the group with internal limiting membrane staining and $0.43 \pm 0.25$ in the group without internal limiting membrane staining $(\mathrm{p}=0.0002)$. We observed improvement of visual acuity in $63 \%$ of cases in the group in which internal limiting membrane staining was performed and in $80.3 \%$ of cases in the group without internal limiting membrane staining. Macular hole closure rates were $76.5 \%$ in the group treated with internal limiting membrane staining and $95.1 \%$ in the group treated without internal limiting membrane staining. Conclusions: Macular hole surgeries followed by internal limiting membrane removal without use of staining have better visual and anatomic results when compared to macular hole surgeries followed by internal limiting membrane removal guided by indocyanine green staining. We recommend caution in using indocyanine green staining in macular hole surgeries due to its possible toxic effect.

Keywords: Macula lutea; Retinal perforations/surgery; Macula lutea/pathology; Indocyanine green; Basement membrane/ surgery; Comparative study

\section{REFERÊNCIAS}

1. Brasil OM. Cistos e forames maculares idiopáticos. In: Lavinsky J, org. Doenças prevalentes da retina e vítreo. Rio de Janeiro: Cultura Médica; 2002. p. $27-35$.

2. Kelly NE, Wendel RT. Vitreous surgery for idiopathic macular holes. Results of a pilot study. Arch Ophthalmol. 1991;109(5):654-9. Comment in: Arch Ophthalmol. 1991;109(5):635-6.

3. Wendel RT, Patel AC, Kelly NE, Salzano TC, Wells JW, Novack GD.
Vitreous surgery for macular holes. Ophthalmology. 1993;100(11):1671-6. Comment in: Ophthalmology. 1993;100(11):1607-8.

4. Eckard C, Eckard U, Groos S, Luciano L, Reale E. [Removal of the internal limiting membrane in macular holes. Clinical and morphological findings]. Ophthalmologie. 1997;94(8):545-51. German.

5. Olsen TW, Sternberg P Jr, Capone A Jr, Martin DF, Lim JI, Grossniklaus HE, et al. Macular hole surgery using thrombin-activated fibrinogen and selective removal of internal limiting membrane. Retina. 1998;18(4):322-9. Comment in: Retina. 1999;19(5):478-9.

6. Brooks HL Jr. Macular hole surgery with and without internal limiting membrane peeling. Ophthalmology. 2000;107(10):1939-48; discussion 1948-9.

7. Kadonosono K, Itoh N, Uchio E, Nakamura S, Ohno S. Staining of internal limiting membrane in macular hole surgery. Arch Ophthalmol. 2000;118(8): 1116-8.

8. Burk SE, Da Mata AP, Snyder ME, Rosa RH Jr, Foster RE. Indocyanine green-assisted peeling of the retinal internal limiting membrane. Ophthalmology. 2000;107(11):2010-4. Comment in: Ophthalmology. 2003;110(9):1864.

9. Brasil OM. Optic nerve atrophy after macular hole surgery. In: Curso avançado do diagnóstico e tratamento das doenças maculares; 2003 Ago 29-30. São Paulo: Hospital Samaritano.

10. Mester V, Kuhn F. Internal limiting membrane removal in the management of full-thickness macular holes. Am J Ophthalmol. 2000;129(6):769-77.

11. Da Mata AP, Burk SE, Riemann CD, Rosa RH Jr, Snyder ME, Petersen MR, et al. Indocyanine green-assisted peeling of the retinal internal limiting membrane during vitrectomy surgery for macular hole repair. Ophthalmology. 2001; 108(7):1187-92. Comment in: Ophthalmology. 2002;109(6):1039-40; author reply 1040-1; Ophthalmology. 2002;109(6):1039; author reply 1040-1; Ophthalmology. 2002;109(6):1040; author reply 1040-1.

12. Kwork AK, Li WW, Pang CP, Lai TY, Yam GH, Chan NR, et al. Indocyanine green staining and removal of internal limiting membrane in macular hole surgery: histology and outcome. Am J Ophthalmol. 2001;132(2):178-83. Comment in: Am J Ophthalmol. 2002;133(4):587-8; author reply 588.

13. Weinberger AW, Schlossmacher B, Dahlke C, Hermel M, Kirchhof B, Schrage NF. Indocyanine-green-assisted internal limiting membrane peeling in macular hole surgery - a follow-up study. Graefes Arch Clin Exp Ophthalmol. 2002;240(11): 913-7. Comment in: Graefes Arch Clin Exp Ophthalmol. 2003;241(7):598; author reply 599-601.

14. Saunders PJR, Nehemy MB, Teixeira MN, Souza EV, Miranda D. Estudo histopatológico de membrana limitante interna removida na cirurgia do buraco macular. Rev Bras Oftalmol. 2002;61(2):87-91.

15. Haritoglou C, Gandorfer A, Gass CA, Schaumberger M, Ulbig MW, Kampik A. Indocyanine green-assisted peeling of the internal limiting membrane in macular hole surgery affects visual outcome: a clinicopathologic correlation. Am J Ophthalmol. 2002;134(6):836-41. Comment in: Am J Ophthalmol. 2003;136(6):1193-4; author reply 1194-6; Am J Ophthalmol. 2003;136(5):961-2; author reply 962.

16. Ando F, Sasano K, Ohba N, Hirose H, Yasui O. Anatomic and visual outcomes after indocyanine green-assisted peeling of the retinal internal limiting membrane in idiopathic macular hole surgery. Am J Ophthalmol. 2004;137(4):609-14. Comment in: Am J Ophthalmol. 2004;137(40):744-6; Am J Ophthalmol. 2004; 138(4):689-90; author reply 690; Am J Ophthalmol. 2004;138(40):690-1; author reply 691; Am J Ophthalmol. 2004;138(4):691-2; author reply 692.

17. Gass CA, Haritoglou C, Schaumberger M, Kampik A. Functional outcome of macular hole surgery with and without indocyanine green-assisted peeling of the internal limiting membrane. Graefes Arch Clin Exp Ophthalmol. 2003;241(9): 716-20.

18. Engelbrecht NE, Freeman J, Sternberg P Jr, AAberg TM Sr, AAberg TM Jr, Martin DF, et al. Retinal pigment epithelial changes after macular hole surgery with indocyanine green-assisted internal limiting membrane peeling. Am J Ophthalmol. 2002;133(1):89-94. Comment in: Am J Ophthalmol. 2002;134(1):150; author reply 151 .

19. Maia M, Haller JA, Pieramici DJ, Margalit E, de Juan E Jr, Farah ME, et al. Retinal pigment epithelial abnormalities after internal limiting membrane peeling guided by indocyanine green staining. Retina. 2004;24(1):157-60. 